\title{
Serviços mínimos de Atenção Primária à Saúde em contextos de crises
}

\author{
Servicios mínimos de atención primaria de salud en contextos de crisis
}

Minimum Primary Health Care services in crisis contexts

\author{
Caique Nogueira de SOUZA ${ }^{(1)}$ \\ Fernanda MANZINI ${ }^{(1)}$ \\ Silvana Nair LEITE ${ }^{(2)}$
}

Recebido: 22 abr 2021

Revisado: 8 maio 2021

Aceito: 9 maio 2021

Autor de correspondência:

Fernanda Manzini

manzinifer@gmail.com

Como citar: Souza CN,

Manzini F, Leite, SN. Serviços

mínimos de Atenção Primária

à Saúde em contextos de

crises. J Manag Prim Health

Care. 2021;13:e06.

https://doi.org/10.14295/jmphc. v13.1138

Conflito de interesses:

Os autores declaram não haver nenhum interesse profissional ou pessoal que possa gerar conflito de interesses em relação a este manuscrito.

Copyright: Este é um artigo de acesso aberto, distribuído sob os termos da Licença Creative Commons (CC-BYNC). Esta licença permite que outros distribuam, remixem, adaptem e criem a partir do seu trabalho, mesmo para fins comerciais, desde que the atribuam o devido crédito pela criação original.

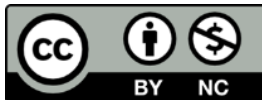

\author{
(1) Prefeitura Municipal de Florianópolis, Florianópolis, SC, Brasil. \\ (2) Universidade Federal de Santa Catarina - UFSC, Departamento de Ciências Farmacêuticas, \\ Florianópolis, SC, Brasil.
}

\section{Resumo}

A Atenção Primária à Saúde se deparou com desafios atípicos durante a pandemia causada pela covid-19, exigindo reorganização de toda a rede de serviços de saúde, que deve manter o cuidado integral e longitudinal dos usuários enquanto preserva saúde de servidores e gerencia recursos. Deste cenário emerge a discussão sobre "serviços mínimos" em períodos de crise no contexto da Atenção Primária à Saúde: o que os define, como priorizá-los e viabilizá-los. Este estudo busca elucidar essas questões através de uma revisão da literatura sobre a organização de serviços de Atenção Primária à Saúde em contextos de crises, bem como o relato e discussão sobre a experiência local na rede de Atenção Primária à Saúde em uma Unidade Básica de Saúde - UBS no município de Florianópolis, SC, durante o enfrentamento da pandemia da covid-19. A pesquisa sobre Serviços Mínimos na Atenção Primária à Saúde apontou escassez de literatura. Propõe-se o conceito de serviços mínimos e, embasado neste, os conceitos de serviços mínimos contexto dependentes e serviços mínimos população dependentes, além da reflexão sobre a organização destes serviços em uma UBS, com destaque para a incorporação de novas tecnologias no atendimento e a gestão do serviço de forma colegiada.

Descritores: Atenção Primária à Saúde; Unidade Básica de Saúde; Gestão em Saúde; Covid-19.

\section{Resumen}

La Atención Primaria de Salud enfrentó desafíos atípicos durante la pandemia causada por covid-19, requiriendo la reorganización de toda la red de servicios de salud, que debe mantener una atención integral y longitudinal a los usuarios preservando la salud de los servidores y la gestión de recursos. De este escenario surge la discusión sobre los "servicios mínimos" en períodos de crisis en el contexto de la Atención Primaria de Salud: qué los define, cómo priorizarlos y hacerlos factibles. Este estudio busca dilucidar estos temas a través de una revisión de la literatura sobre la organización de los servicios de Atención Primaria de Salud en contextos de crisis, así como el informe y discusión sobre la experiencia local en la red de Atención Primaria de Salud en una capital brasileña durante el enfrentamiento al covid-19 pandemia. La investigación sobre Servicios Mínimos en Atención Primaria de Salud señaló una escasez de literatura. Se propone el concepto de servicios mínimos y, en base a este, los conceptos de servicios mínimos dependientes del contexto y servicios mínimos 
ISSN 2179-6750

dependientes de la población, además de la reflexión sobre la organización de estos servicios en una Unidad Básica de Salud, con énfasis en la incorporación de nuevas tecnologías en el servicio y en la gestión del servicio de manera colegiada.

Palabras-claves: Atención Primaria de Salud; Centros de Salud; Gestión em Salud; Covid-19.

\begin{abstract}
Primary Health Care faced atypical challenges during the covid-19 pandemic, requiring a reorganization of the entire network of health services, which must maintain comprehensive and longitudinal care for users while preserving the health of servers and managing resources. From this scenario emerges the discussion about "minimum services" in periods of crisis in the context of Primary Health Care: what defines them, how to prioritize and make them feasible. This study seeks to elucidate these issues through a literature review on the organization of Primary Health Care services in crisis contexts, and the report and discussion based on the local experience in the Primary Health Care network in a Brazilian capital during the coping with the covid-19 pandemic. The research on Minimum Services in Primary Health Care pointed out a lack of literature. The concept of minimum services is proposed and, based on this, the concepts of context-dependents minimum services and population dependent minimum services, in addition to the reflection about the organization of these services in a Primary Care Health service, with emphasis on the incorporation of new technologies in the service and management of the service in a collegiate manner.
\end{abstract}

Keywords: Primary Health Care; Health Centers; Health Management; Covid-19.

\title{
Introdução
}

A Atenção Primária à Saúde - APS vem se consolidando como alicerce do Sistema Único de Saúde - SUS, constituindo-se de uma plêiade de cuidados essenciais de saúde, embasados cientificamente e adequados socioculturalmente, de alcance universal, estabelecendo-se como primeiro nível de contato do indivíduo, da família e da comunidade com o sistema, sendo o elemento inicial de um processo continuado de assistência à saúde. ${ }^{1,2}$

Em março de 2020, a Organização Mundial da Saúde - OMS anunciou a situação pandêmica de covid-19, doença emergente causada pelo vírus Sars-Cov-2 (Severe Acute Respiratory Syndrome Coronavirus 2). ${ }^{3}$ Em poucos meses todo o globo deparou-se a uma crise de saúde pública de magnitude não vista há pelo menos 100 anos, comparável à gripe espanhola de 1918, porém de comportamento e desfechos potencialmente mais drásticos, graças à redução do tempo-espaço que tornou o mundo do século XXI em uma "aldeia global", como previsto por Marshall McLuhan. ${ }^{4}$

Frente a esse contexto atípico, a APS enfrenta o desafio de seguir as recomendações de distanciamento social - evitando aglomerações e diminuindo atendimentos presenciais em seus serviços. Ao mesmo tempo, precisa organizar-se frente à redução de recursos humanos por conta dos afastamentos de trabalhadores em grupos de risco ou adoecidos e a gestão de insumos (equipamentos de proteção individual em especial) para garantir a segurança de seus profissionais, mantendo "o enfoque mais eficaz, eficiente e equitativo para melhorar a saúde". ${ }^{5}$ 
Em 2019, o Ministério da Saúde lançou a Carteira de Serviços da Atenção Primária à Saúde com o intuito de definir e nortear o escopo das ações das unidades de saúde da APS, ${ }^{6}$ seguindo as orientações contidas na Política Nacional de Atenção Básica. No entanto, apesar do importante suporte oferecido no documento em tempos de normalidade, a APS se deparou com questões, de certa forma, inéditas, provindas da situação excepcional criada pela pandemia da covid-19, que no caso brasileiro foram agravadas pela incoordenação de ações entre o governo federal e as autoridades estaduais e locais. ${ }^{7}$

Este cenário de excepcionalidade gera angústia em trabalhadores e gestores da APS. O motivo para tal é a ausência de uma definição clara do que seriam os serviços mínimos da Atenção Primária à Saúde - ou seja, em tempos de crise, quais serviços seriam considerados indispensáveis. Outra fonte de inquietação, tão desafiadora quanto saber quais são os serviços mínimos, é o modo como organizar e viabilizar a realização dos mesmos. Este último impasse, dada à excepcionalidade do momento, exigiu (e ainda exige) inovações, exemplificado pela incorporação de ferramentas como a telemedicina, regulamentada por portaria ministerial, inclusive ampliando seu escopo de atuação para maior resolubilidade no enfrentamento da pandemia da covid $-19 .{ }^{8}$

Em que pese as dificuldades do momento e escassez de literatura sobre a problemática, este trabalho objetiva construir a discussão do tema e a proposição de conceitos para embasar as práticas nos serviços de Atenção Primária à Saúde, inclusive em outros momentos de crise, sejam por epidemias ou por tensões sociopolíticas, valendo-se da experiência vivenciada na pandemia de covid-19 em uma Unidade Básica de Saúde - UBS no município de Florianópolis (SC).

\section{Metodologia}

Realizou-se uma revisão da literatura, investigando a existência de uma definição consistente do conceito de serviços mínimos de forma geral e, em seguida, o recorte de sua aplicação à APS. Para tanto, inicialmente, levantou-se textos teórico-conceituais em português, espanhol e inglês, que discorriam sobre serviços mínimos de maneira ampla.

A definição dos conceitos de serviços mínimos e os derivados deste foi realizada considerando os apontamentos da literatura obtidos na revisão, associados ao vivenciado durante a pandemia de covid-19.

O relato de experiência acerca da reorganização da APS em Florianópolis, em particular de uma UBS do Distrito Centro, fundamentou-se na vivência dos autores no atendimento dos usuários por uma equipe de saúde da família, dos atendimentos realizados na sala de sintomáticos respiratórios e na gestão do serviço. 
O trabalho foi aprovado pelo Comitê de Ética em Pesquisa com Seres Humanos da UFSC (CAAE: 35661220.7.0000.0121).

\section{Resultados}

\section{O enfrentamento da COVID-19 em uma Unidade Básica de Saúde}

A UBS aqui descrita é uma das unidades de APS da cidade de Florianópolis, referência para atendimento de dois bairros, localizada entre as regiões central e norte da ilha. Composta por 7 Equipes de Saúde da Família - ESF, além de profissionais do Núcleo de Apoio à Saúde da Família - NASF e administrativos, responsáveis pelo atendimento de uma população adscrita de aproximadamente 19 mil pessoas, segundo estimativas de 2015 (baseadas no Censo de 2010). O território abarca diversas áreas de interesse social, mas também regiões de classe média, com intenso dinamismo e diversidade de demandas em seu cotidiano, um dos motivos pelos quais estabeleceu-se como cenário de ensino para graduandos e residentes de diversas categorias profissionais da saúde.

Um dia após a declaração de pandemia por Sars-CoV-2 pela OMS, ${ }^{3}$ em 12 de março, os dois primeiros casos de COVID-19 foram confirmados em Florianópolis, exigindo pronta reorganização dos serviços de saúde.

Apesar do enfoque individualista adotado pelas autoridades nacionais, baseando-se unicamente em criação de leitos de UTI, respiradores e hospitais de campanha, a gestão da Secretaria de Saúde e os trabalhadores reconheceram prontamente que o nó crítico para o controle do contágio e mitigação dos efeitos da epidemia se daria no âmbito da APS, única estratégia capaz de unificar a assistência individual às medidas comunitárias, como destacado por Giovanella et al. ${ }^{9}$

Em resposta quase que imediata, apoiando-se nos princípios de autonomia e gerenciamento local, há apenas um dia da divulgação dos primeiros casos na cidade, reuniram-se médicos, enfermeiros e coordenação da UBS a fim de determinarem-se as primeiras ações e mudanças a serem realizadas no serviço. As questões elencadas como prioritárias no momento estão apresentadas no Quadro 1.

Quadro 1. Questões elencadas como prioritárias no momento pandêmico para reorganização dos serviços

Questões prioritárias elencadas para reorganização do serviço

Estratégia de triagem de usuários sintomáticos respiratórios;

Definição da equipe de atendimento da sala de usuários sintomáticos respiratórios (USR);

Alocamento racional de Equipamentos de Proteção Individual (EPI);

Estratégias para diminuição da circulação de usuários na UBS; 
Questões prioritárias elencadas para reorganização do serviço

Atendimentos de pacientes não USR que deveriam ser mantidos presencialmente;

Atendimentos de pacientes não USR que poderiam ser realizados de forma remota e a definição de qual a forma de atendimento remoto a ser utilizada;

Atendimentos eletivos que poderiam ser postergados;

Reorganização das funções das diversas categorias profissionais na UBS, incluindo o NASF;

Afastamento de servidores pertencentes a grupos de risco;

Manutenção de serviços como: sala de vacinas, sala de curativos, dispensação de medicamentos, procedimentos eletivos, visitas domiciliares e grupos terapêuticos.

Fonte: Elaborado pelos autores (2020).

Emerge, então, implicitamente, a discussão sobre serviços mínimos. Doravante neste trabalho, analisar-se-á esses pontos, ponderando sobre como as ações adotadas durante o contexto crítico da pandemia de 2020 dialogam com a construção empírica das ideias de serviços mínimos na APS.

\section{"Serviços Mínimos" e "Serviços Essenciais"}

Dois fenômenos semânticos, a polissemia (vários sentidos para uma mesma palavra) e a sinonímia (palavras distintas com mesmo significado) contribuem para o emprego de vocábulos distintos para exprimir os mesmos conceitos, o que por vezes, gera confusão e distorce a ideia originalmente concebida. Destarte, advém a necessidade de definir uma terminologia mais precisa, que cumpra seu papel, segundo defende Cabré, ${ }^{10}$ como forma de transmissão e comunicação dentro das disciplinas técnico-científicas. Os termos serviços mínimos e serviços essenciais são exemplos de expressões técnicas utilizadas corriqueiramente, até mesmo em textos especializados, de maneira imprecisa e pouco conceitualizada, não incomumente como sinônimos. Segundo o Dicionário Caldas Aulete, um dos significados para essencial (https://www.aulete.com.br/essencial) é o que constitui a essência, o básico ou fundamental de algo, enquanto não há correlato no verbete (https://www.aulete.com.br/minimo) referente à palavra mínimo na mesma referência, rompendo com os requisitos para total sinonímia propostos por Lyons.

A pesquisa por definições de serviços mínimos e serviços essenciais, em contextos gerais, é árida, o que corrobora para o uso pouco criterioso desses termos. Contudo, há na literatura jurídica e legislativa o uso dessas expressões com significados diferentes entre si, oferecendo subsídios para melhor distinção dos seus conceitos subjacentes. $O$ texto da Lei n. 7.783, de 28 de junho de 1989, que dispõe sobre o exercício do direito à greve no Brasil, se refere a "serviços ou atividades essenciais", entre eles a assistência médica e hospitalar, porém não há menção ao termo "serviço mínimo" na carta. Em Portugal, a lei que trata do 
direito à greve é a Lei n. 65/77, de 26 de agosto, a partir da qual a Procuradoria Geral da República elaborou um parecer requerido pelo Ministério da Saúde português, datado de 1990, apresentando em suas conclusões as duas expressões, simultaneamente, com sentidos distintos.

4 - Durante a greve nos serviços públicos essenciais, como os serviços de saúde, médicos e hospitalares, deve ser assegurada pelas associações sindicais e pelos trabalhadores em greve a prestação de serviços mínimos indispensáveis à satisfação das necessidades sociais fundamentais. ${ }^{11}$

Consoante ao uso do termo "serviços essenciais" pela jurisprudência portuguesa citada acima, o Decreto n. 10.282, de 20 de março de 2020, da Presidência da República do Brasil, que regulamentou a Lei n. 13.979 - a qual dispõe sobre o enfrentamento à emergência sanitária da COVID-19 - utiliza-se em seu texto da expressão atividades essenciais, não havendo, contudo, o uso do termo serviços mínimos na redação.

$\S 1^{\circ}$ São serviços públicos e atividades essenciais aqueles indispensáveis ao atendimento das necessidades inadiáveis da comunidade, assim considerados aqueles que, se não atendidos, colocam em perigo a sobrevivência, a saúde ou a segurança da população, tais como:

I - assistência à saúde, incluídos os serviços médicos e hospitalares. ${ }^{12}$

Embasado pelo emprego dos termos pela legislação e jurisprudência, infere-se que serviços essenciais melhor descreve um gênero de atividades, vinculadas entre si por seu impacto na população, sem as quais a manutenção da sociedade é ameaçada. A classificação dos serviços de saúde como atividades essenciais é axiomática, pela obviedade dos efeitos deletérios que podem advir da falta de acesso da comunidade à assistência à saúde. Paralelamente, a Atenção Primária à Saúde, como definiu a declaração de Alma-Ata, é constituída por cuidados essenciais em saúde, fato reafirmado durante a pandemia da infecção pelo Sars-CoV-2, apontando-se, segundo a OMS, como uma "fundação essencial para a resposta global à COVID-19", ${ }^{13, \text { p. } 1}$ o que ratifica as conclusões de Starfield. ${ }^{14}$

Por sua vez, o termo serviços mínimos aplicar-se-ia de forma mais consistente ao conjunto de funções específicas desenvolvidas pelas referidas atividades essenciais que, sob circunstâncias adversas, devem ser priorizadas e oferecidas aos usuários. Retomando a alusão ao contexto de greve, a legislação brasileira não versa sobre quais seriam os serviços mínimos de cada atividade essencial, mas prevê que sejam fixados limites percentuais mínimos para suprir as necessidades inadiáveis da população, sendo omissa, no entanto, em 
ISSN 2179-6750

determinar qual é a margem percentual nestes casos, ficando esta tarefa a cargo do Poder Judiciário, como analisado por Batista. ${ }^{15}$ Segundo a mesma autora, a definição dos serviços mínimos tem como objetivo manter as atividades cuja interrupção durante um movimento grevista, por exemplo, possa comprometer a subsistência da sociedade, ou seja, é um subconjunto das ações desempenhadas pelos serviços essenciais.

Sopesando as confusões oriundas dos fenômenos semânticos e as lacunas conceituais quanto às expressões "serviços mínimos" e "serviços essenciais", para fins de clareza terminológica e desambiguação dos termos, são propostas as seguintes definições (Quadro 2):

Quadro 2. Definição de serviços ou atividades essenciais e serviços mínimos

Conceito

Definição

Serviços ou atividades Classe de atividades de relevância para a sociedade em determinado período,

essenciais

Serviços mínimos cuja ausência incorra em perigo para a sua manutenção.

Conjunto de funções desempenhadas pelas atividades essenciais que devem ser priorizadas em contextos de crise, a fim de suprir as necessidades inadiáveis da população.

Fonte: Elaborado pelos autores (2020).

Destaca-se nas definições propostas acima que o conceito de serviços mínimos deve ser ligado às condições de ruptura da normalidade do exercício das atividades essenciais, em particular dos serviços de saúde, como a APS. Tal pressuposto se justifica na evitação de uma racionalidade empobrecida do oferecimento desses serviços essenciais, nivelada pelo fazer o mínimo possível, contrária aos ideais de otimização e da integralidade da atenção, e às iniciativas de incentivo ao aprimoramento contínuo do serviço público. De tal forma, os serviços mínimos da APS emergem como constructos cuja inalidade é oferecer a melhor assistência possível durante momentos de crise, ideia divergente dos comumente chamados Pacotes Mínimos/Básicos de Saúde (Minimum Services Packages), que propõe o estabelecimento de um grupo de serviços de saúde que sejam minimamente suficientes para determinada população (principalmente de países/localidades empobrecidas).

\section{Serviços Mínimos Contexto Dependentes - SMCD}

O atendimento ao paciente com sintomas respiratórios tornou-se uma prioridade indiscutível durante a pandemia da covid-19, contudo, devido ao comportamento da 
epidemia na comunidade e as ações necessárias para contê-la, diversos aspectos suscitaram debates cujas conclusões não se encontravam, a priori, sob o horizonte da obviedade.

Foge do escopo deste trabalho a discussão aprofundada sobre protocolos de atendimento aos usuários sob suspeita de infecção pelo Sars-CoV-2 na APS. No entanto, a experiência adquirida ao longo do curso da pandemia propicia reflexões acerca da capacidade de adaptação dos serviços de atenção primária frente a condições adversas.

Primeiramente, fez-se necessária realização de triagem de todos os pacientes e acompanhantes, rastreando sintomas, sinais e comportamentos (contato com casos suspeitos ou confirmados para covid-19), antes mesmo de adentrarem na UBS, para minimizar a circulação de potenciais portadores, sintomáticos ou não, do novo coronavírus em áreas destinadas ao atendimento de outros quadros, devendo, aqueles sob suspeita, serem encaminhados a espaço separado (chamada de Sala de Atendimento aos usuários sintomáticos respiratórios - USR ou Sala USR) para devidas avaliação e conduta. Mesmo esta medida inicial, apoiada pelas orientações da gestão municipal de saúde, apresentou repercussões sobre a estruturação dos serviços em toda unidade.

Ao se estabelecer a necessidade de novas funções dentro de uma unidade de saúde (como triagem e Sala USR), sem o concomitante aumento do quadro de funcionários - em realidade com restrição de recursos humanos, em vista dos afastamentos dos trabalhadores sintomáticos e pertencentes a grupos de risco - implica, inevitavelmente, em redução de pessoas disponíveis para o desempenho de outras funções, podendo, inclusive, inviabilizar a realização de algumas dessas atividades. Tal decisão é revestida de muitas incertezas e de alto grau de responsabilidade, pois qualquer serviço que fique em condições subótimas no âmbito da APS pode, em maior ou menor proporção, levar a agravamentos na saúde da comunidade sob sua assistência.

Cabe, então, sopesar qual a real necessidade de estabelecerem-se novas tarefas em um contexto crítico, isto é, em um cenário de restrição absoluta ou (acentuamento) das restrições relativas de recursos, qual a urgência, impacto e viabilidade da criação de novas atividades. Em exemplo, em um contexto de greve, a criação de um setor de triagem pode ser adequada para que não se impeça o acesso de pessoas com quadros potencialmente graves, por outro lado, não estando em período epidêmico, a formação de uma equipe específica para atendimento unicamente de quadros respiratórios não é justificada.

Observa-se que existe um grupo de funções cuja manutenção ou desenvolvimento em momentos adversos não é universal, mas sim definida conforme as demandas prioritárias apresentadas aos serviços nesses períodos. Denominar-se-á este grupo de serviços mínimos 
contexto dependentes (SMCD), definidos como aqueles que devem ser desempenhados em contextos críticos a depender do cenário de base e que contemplem os seguintes critérios:

- Necessidade;

- Urgência;

- Alto impacto presumido (principalmente em desfechos duros, tais como: mortalidade geral e por causa específica, internações hospitalares etc.);

- Viabilidade.

Em consonância com a definição exposta, durante a pandemia, emergiram como grupo de SMCD, as seguintes atividades: triagem, sala USR, monitoramento de casos e testagem (realização de testes rápidos para covid-19 na UBS).

Após elencados os SMCD, faz-se mister que se desenvolvam estratégias para a sua disponibilidade, além da reavaliação contínua dos parâmetros que os sustentam, pois em decorrência do dinamismo próprio das crises, em dado momento, novas funções podem se apresentar como SMCD, enquanto outras podem de deixar de sê-los, incitando perspicácia dos gestores e flexibilidade do serviço de saúde para adaptar-se adequadamente às mudanças que, inexoravelmente, ocorrerão ao longo de um período de instabilidades.

\section{Serviços Mínimos População Dependentes - SMPD}

Um dos atributos essenciais da APS é a atenção ao primeiro contato, que abrange, como explanado por Oliveira e Pereira, ${ }^{16}$ os conceitos de acesso e acessibilidade, sendo o primeiro estruturado na minimização das restrições de entrada aos serviços de saúde e à percepção do usuário sobre a facilidade de busca por assistência e resolução de suas demandas, enquanto o segundo refere-se à maneira que os serviços são ofertados e sua capacidade resolutiva.

Corolário ao atributo de primeiro contato, estabelece-se a Atenção Primária como porta de entrada do sistema de saúde, o que, associado à sua função como coordenadora dos cuidados dos indivíduos e famílias na Rede de Atenção à Saúde - RAS, torna-a diretamente responsável pela saúde da população sob seus cuidados, responsabilidade esta que não pode ser negligenciada durante decisões que visem a reorganização de suas atividades, especialmente em períodos atípicos.

Como discorrido anteriormente, ao deparar-se frente a um momento disruptivo, por vezes, os serviços de APS precisarão redirecionar recursos para a realização de funções necessárias para o enfrentamento das demandas impostas pelo contexto em questão - os SMCD. No entanto tal movimento não é inócuo, pois subtrai capital humano e material de outras atividades, salvo nos casos de recebimento de subsídios para a execução dessas tarefas, situação excepcional na experiência da maioria das unidades de saúde. 
ISSN 2179-6750

De tal maneira, depois de serem definidos os SMCD, o próximo passo é o esclarecimento de quais outros serviços devem ser priorizados a fim de se evitar desfechos desfavoráveis (principalmente a curto e médio prazo) na saúde da comunidade. Esta etapa pode mostrar-se mais desafiadora que a primeira, considerando-se que a complexidade da APS se deve não apenas a diversidade clínica das necessidades em saúde que lhe são demandadas, mas também aos problemas psicológicos e sociais que contribuem para o adoecimento da população.

Durante a pandemia da covid-19 foram publicados alguns documentos visando o alinhamento das atividades dos serviços de APS, como o divulgado pelo Conselho Nacional dos Secretários de Saúde ${ }^{17}$ e, no caso do município de Florianópolis, a Comunicação Interna Circular 04 da Gerência de Atenção Primária, datada de 14/07/2020. É inegável a importância de tais textos orientadores, mas ainda assim muitas UBS podem encontrar dificuldades para atender a todas as suas recomendações, em especial quando vivenciam redução acentuada do quadro de servidores, aumento da demanda assistencial acima da capacidade previamente instalada ou outras questões idiossincráticas que não são contempladas pelas redações normativas.

Fundamenta-se que, a partir do reconhecimento das necessidades principais da população adscrita a seu território (ou lista de pacientes), os serviços de APS estabeleçam o grupo de ações cuja continuidade é imprescindível, o qual denominar-se-á de Serviços Mínimos População Dependentes - SMPD. Ressalta-se que a escolha da terminologia parte da ideia de que, apesar destes serviços não serem vinculados a um contexto de crise específico, os são ao contexto epidemiológico, social e cultural da população assistida, não sendo de todo adequado o uso do termo "Contexto Independente", como poder-se-ia intuir a fim de conotar contraste aos já definidos SMCD.

Cabe atentar que os SMCD e os SMPD não são conceitos mutuamente excludentes, tampouco de oposição, considerando-se que, a depender do cenário em questão, as necessidades emergentes do contexto e as requeridas basalmente pela população podem se sobrepor. Em exemplo, suponha-se um período de profunda recessão econômica em uma comunidade já vulnerabilizada, neste caso o acesso à assistência social e a vigilância territorial de violação de direitos encontrar-se-ão contemplados pelas definições de SMCD e SMPD simultaneamente.

Definir a lista de SMPD é tarefa de grande complexidade, pois mesmo equipes que já estão há muito sintonizadas com as necessidades de seu território e/ou lista de pacientes podem ter dificuldade de delinear exatamente qual o real impacto que certos serviços têm na saúde da população em períodos de crise. Ilustrativo desse processo foi o movimento adotado por muitos serviços de APS, durante a pandemia, de aprazar o seguimento de rotina 
ISSN 2179-6750

de pacientes multi-comórbidos e que se apresentavam há tempos em relativa estabilidade clínica, com o intuito inicial de preservá-los de uma possível exposição ao Sars-CoV-2 dentro das UBS, bem como para direcionar os recursos à assistência do crescente número usuários acometidos pela COVID e/ou outras condições agudas. No entanto, tal atitude corroborou para o agravamento de muitos desses usuários, fazendo com que várias equipes, principalmente no último trimestre do ano, presenciassem o que foi denominado de Terceira Onda por Mendes, ${ }^{18}$ um fenômeno que, apesar de previsto e esperado que ocorresse - e continue ocorrendo - em todo o mundo, abala profundamente famílias e profissionais.

Outra situação emblemática foi a queda dramática da cobertura vacinal em todo território nacional, questão que já vinha preocupando autoridades sanitárias nos últimos anos, devido em parte aos movimentos anti vacinas, mas que foi potencializada pelo temor do contágio pela covid-19 em 2020, levando as taxas de vacinação a níveis alarmantes. $\mathrm{Na}$ realidade da UBS, logo ao início da pandemia, muito discutiu-se sobre a viabilidade de manter o funcionamento da sala de vacinas que, como em outras grandes unidades de APS, é um setor de grande circulação de pessoas, principalmente crianças, grupo inicialmente suspeito como potenciais vetores do Sars-CoV-2, dado as altas porcentagens de infecções assintomáticas na população pediátrica. Contudo, optou-se pela manutenção do serviço, considerando-o como um SMPD, ainda que para tanto precisasse ser adaptada a sua forma de atendimento, como será abordado mais adiante.

Ainda que não haja referências bem estabelecidas e totalmente aplicáveis às realidades locais sobre os SMPD, alguns conceitos já conhecidos podem subsidiar as decisões sobre o tema. Em relação às atividades de assistência e prevenção de quadros clínicos, uma das publicações que podem embasar a escolha dos SMPD é a Portaria n. 221, do Ministério da Saúde, ${ }^{19}$ que define a lista brasileira de Condições Sensíveis à Atenção Primária, ou seja, as patologias cujos índices de ocorrência e/ou agravamento são vinculados ao trabalho da APS e, por tanto, seria prudente a manutenção daqueles serviços que as contemplem.

Todavia, principalmente em momentos de crise, em que potencialmente se agravam as iniquidades sociais e os quadros de sofrimento psíquico, é importante que se atente para

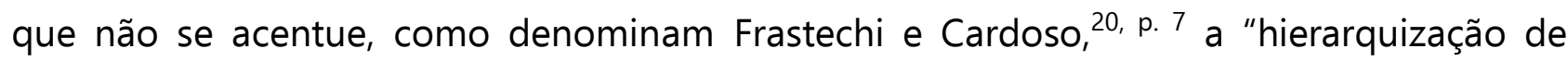
saberes", levando à precarização dos cuidados em saúde mental . Em especial nas situações de isolamento social e de quarentena, como na pandemia da covid-19, problemas como comportamento suicida e violência doméstica, que já assolam a sociedade em momentos de "normalidade", tornam-se ainda mais preocupantes, tanto pelos indícios de real elevação desses casos, quanto pela dificuldade de vigilância territorial nesses tempos. 
ISSN 2179-6750

Depreende-se, então, que a definição dos SMPD exige profundo conhecimento demográfico, epidemiológico e sociocultural do território e/ou lista de pacientes, bem como a conscientização sobre as responsabilidades das unidades de atenção primária sobre a saúde física, mental e social da população sob seus cuidados, para que seja feita a alocação adequada dos recursos disponíveis e que se preserve, dentro das possibilidades do momento, a acessibilidade e o acesso em contextos de crise. O Quadro 3 apresenta a comparação entre os SMCD e os SMPD:

\section{Quadro 3. Comparação entre os conceitos de SMCD e SMPD}

Serviços Mínimos Contexto Dependentes (SMCD) Dependem das causas e repercussões das crises; Características fundamentais: Necessidade Urgência, Alto impacto e Viabilidade; Recursos são primeiramente realocados para sua execução.
Serviços Mínimos População Dependentes (SMPD) Dependem das características epidemiológicas e socioculturais da população; Podem se sobrepor aos SMCD;

Planejamento leva em conta os recursos disponíveis após definição dos SMCD.

Fonte: Elaborado pelos autores (2020).

\section{A execução dos serviços mínimos da APS em contextos de crise}

Depois de estabelecidos quais são os serviços mínimos (SMCD e SMPD) em uma UBS, o próximo passo é definir como os mesmos serão executados. Nesta etapa, duas máximas devem guiar o pensamento dos gestores: otimização dos recursos e inovação das práticas. Para tanto, é preciso que todos os atores do processo estejam cientes que este planejamento se alicerça no terreno das incertezas, devendo-se considerar logo de início a capacidade de mudanças das ações frente a alternâncias inesperadas, sem que se perca o foco na resolução dos problemas com respostas realistas, adotando-se uma postura de Planejamento Estratégico Situacional, como explanado por Lacerda, Botelho e Colussi, ${ }^{21}$ considerando as necessidades em saúde.

Congruente aos pensamentos de otimização dos recursos, as unidades de saúde precisam encontrar novas formas de desempenhar suas atividades, inovando tanto nos serviços, processos, comunicação/marketing e, principalmente, no nível organizacional, como identificado por Nodari et al., ${ }^{22}$ ao analisarem as práticas inovadoras da APS em um município do Rio Grande do Sul.

Durante a pandemia da covid-19, o uso das ferramentas de tecnologias de comunicação tornou-se componente cotidiano das atividades dos serviços de APS, especialmente após a regulamentação da telemedicina durante a pandemia pelo Ministério da Saúde, o que permitiu a realização de atendimentos sem expor profissionais e pacientes a riscos desnecessários de contaminação pelo coronavírus. Porém, as equipes de saúde, em 
ISSN 2179-6750

pouco tempo, reconheceram outras potencialidades das ferramentas digitais para além de teleatendimentos. Na UBS, por exemplo, o uso de agendamentos de imunizações por WhatsApp possibilitou o funcionamento da sala de vacinas de forma mais segura e ágil, eliminando as filas de espera e, por se saber com certa antecedência quais e quantas vacinas seriam aplicadas, permitiu melhor controle e programação da reposição de insumos, diminuindo tanto o desperdício quanto a falta dos mesmos.

Outro exemplo de inovação e otimização de recursos propiciada pela tecnologia é o serviço de atendimento pré-clínico "Alô Saúde Floripa", que já estava em processo de organização antes da pandemia e, nesta, apresentou-se como um aliado dos serviços de saúde, auxiliando no direcionamento dos usuários dentro dos fluxos da rede e nas tomadas de decisão, por meio de atendimentos remotos aos pacientes via telefone, aplicativo de celular e website. ${ }^{23}$ Assim, rapidamente a população se apropriou do uso das ferramentas digitais como forma de acesso aos serviços de saúde, muitas vezes preferindo-o em relação à modalidade presencial.

As mídias digitais também abriram espaço para inovações de marketing, seguindo a nomenclatura usada por Nodari et al., ${ }^{22}$ como forma de comunicação dinâmica dos serviços de saúde com a comunidade e também de educação em saúde. Em exemplo, os residentes da UBS criaram vídeos de esclarecimentos de temas correlacionados à covid-19 e ao funcionamento da UBS, que foram publicados nas páginas oficiais da unidade e compartilhados via listas de transmissão do WhatsApp para pacientes, buscando combater notícias falsas e atualizar a população sobre a situação da pandemia na comunidade.

Ainda que, de forma geral, o uso das tecnologias de informação e comunicação apresenta-se com saldo positivo, por ser relativamente recente seu uso na saúde em nosso país, alguns aspectos ainda precisam ser aprimorados. Uma das situações que geraram preocupações por parte de alguns profissionais foi o fato verificado de que, pela modalidade remota, grande parte das solicitações eram realizadas por pessoas mais jovens e, muitas das vezes, com necessidades de saúde de natureza benigna e autolimitada, enquanto grupos que não tinham acesso às tecnologias e/ou alfabetização digital - em sua maioria composta por idosos ou pessoas vulnerabilizadas socialmente - poderiam encontrar dificuldade para acessar os serviços de saúde. A fim de se evitar a Lei dos Cuidados Inversos, descrita em 1971 por Julian Tudor Hart, na qual os recursos de saúde são menos utilizados por aqueles que mais os necessitam, e orientadas pelo princípio da equidade, algumas equipes começaram o trabalho de identificar usuários com dificuldades para o uso de tecnologias, garantindo o acesso destes via buscas ativas e priorização dos atendimentos presenciais. ${ }^{24}$

Outro setor que se mostrou estratégico durante a pandemia foi o da assistência farmacêutica. Na UBS uma das ações implementadas a fim de diminuir a circulação de 
pessoas pela unidade foi a descentralização de alguns medicamentos (como analgésicos comuns, anti-inflamatórios, antieméticos e sais de reidratação oral) da farmácia para os consultórios, além de disponibilizar antibióticos, corticoides, soro para lavagem nasal e bronco dilatadores na sala de atendimento aos usuários sintomáticos respiratórios, com o controle da dispensação das receitas via sistema de comunicação interna. Ainda, começouse a aceitar receitas digitais geradas nos teleatendimentos, a aumentar a quantidade dispensada de remédios de uso contínuo e, até mesmo, realizar teleconsultas farmacêuticas para orientar pacientes sobre uso adequado dos medicamentos. Também, por meio da vigilância farmacêutica, auxiliaram as equipes a identificar usuários que não completavam esquemas de tratamento de interesse epidemiológico, como os casos de sífilis que, dada a alta demanda decorrente dos casos de covid-19, por vezes escapavam do monitoramento feito pelas próprias equipes de saúde da família.

O atendimento presencial ao usuário sintomático respiratório, realizado na Sala USR, também foi um cenário de inovação organizacional na UBS, por meio de uma modalidade de atendimento sincrônico, em que dois profissionais - um médico e um enfermeiro atendem simultaneamente o mesmo paciente, a fim de confluir saberes e habilidades, tornando o atendimento mais ágil e completo. Este modelo de atendimento, desenvolvido e aperfeiçoado inicialmente por dois profissionais da própria UBS, se mostrou facilmente reprodutível pelos pares, surgindo como um método promissor de atendimentos de quadros específicos em situações epidêmicas. Paralelamente, a UBS optou por tornar a realização de Testes Rápidos para Sars-CoV-2 um serviço separado dos demais, com escala própria de profissionais e lista de agendamento de horários, inclusive realizando um evento de testagem em massa, utilizando o espaço de uma escola do bairro, durante um dos picos de contágio pelo vírus na comunidade.

Toda a reorganização do serviço foi pactuada e discutida com as instâncias do controle social. O Conselho Local de saúde da UBS é atuante e tem a presença de diversas lideranças dos bairros do território de abrangência. Mesmo durante a pandemia as reuniões plenárias (mensais) foram mantidas, de maneira on-line, e contaram com a presença de trabalhadores e da população, tendo sempre a temática da pandemia como uma das pautas. A plenária on-line permitiu uma ampliação da participação da população e deve ser uma estratégia a ser adotada mesmo após a pandemia.

As experiências vivenciadas durante a pandemia da covid-19 demonstram a multiplicidade de formas que os serviços de APS podem efetuar as suas atividades em momentos críticos, seja reestruturando processos que já existiam ou mesmo criando soluções inéditas, de maneira que se assegure a disponibilidade dos SMCD e SMPD. Todas essas ações, desde reconhecer a crise, elencar quais são os serviços mínimos, contexto e/ou 
ISSN 2179-6750

populações dependentes, até a execução das tarefas necessárias, exigem alta capacidade de planejamento e reavaliação constante, incumbência hercúlea mesmo para o mais experiente dos gestores. Resta, então, discutir como as UBS podem se organizar nestes cenários.

\section{A organização dos serviços mínimos na APS em contexto de crise: o papel do colegiado gestor}

Gerenciar as atividades de um serviço de APS é tarefa ordinariamente complexa, que exige diversas competências, como elucidam André e Ciampone. ${ }^{25}$ Todavia, em contextos de crise, o nível de complexidade para as tomadas de decisão se aprofunda, determinando que os gestores reestruturem a própria forma de se fazer gestão. Neste sentido, Cecílio ${ }^{26}$ caracteriza a gestão colegiada como alternativa aos modelos gerenciais hegemônicos, contribuindo para processos inovadores, desde 1980, em todos os níveis de saúde.

O Colegiado Gestor (ou Colegiado de Gestão), espaço onde se concretiza a gestão colegiada, ${ }^{26}$ é composto por representantes de diversas categorias profissionais e equipes de saúde e possui a função não apenas de discutir o funcionamento da unidade de saúde, mas principalmente de deliberar, discutindo, planejando e definindo as ações e organização dos serviços na unidade, em consonância com a gestão participativa via Conselho Local de Saúde. A UBS há anos conta com as atividades do Colegiado Gestor, de periodicidade semanal, com a participação de um representante de cada ESF e de categorias profissionais diversas, além da coordenação local, sendo que em ocasiões excepcionais se convoca o chamado "Colegiado Gestor Ampliado", com a participação de mais representantes para o debate e estabelecimento de situações mais complexas e/ou específicas.

Durante a pandemia da covid-19, a gestão colegiada assumiu papel de suma importância no planejamento estratégico da unidade. Segundo a percepção do autores, o ambiente propiciado pelo Colegiado Gestor é o mais adequado para a definição e deliberação sobre os serviços mínimos em contextos críticos, pela inviabilidade de tema tão complexo ser gerenciado unicamente pela coordenação local, enquanto que nas reuniões gerais - nas quais todos os trabalhadores da UBS estão presentes - temas que demandam resoluções práticas, em geral, podem se dispersar em discussões circulares, muitas vezes sem consenso entre as partes, sendo preferível a utilização destes espaços para o compartilhamento de informações, educação continuada e alinhamento de condutas.

Aspecto importante a ser ressaltado é que as atividades do Colegiado Gestor não prescindem das orientações e deliberações advindas dos níveis centrais de gestão, seja municipal, estadual ou federal, mas sim, exercendo a autonomia da gestão local, adequam tais recomendações à realidade da comunidade e do centro de saúde. Tampouco cerceia- 
ISSN 2179-6750

se o trabalho do coordenador da unidade, que deve, imprescindivelmente, ser participante do colegiado, no qual pode externalizar seus posicionamentos e preocupações gerenciais, atentando-se para não se impor de forma autoritária, o que romperia com a razão de ser do colegiado gestor, cujo princípio é justamente a cogestão.

Considerando-se o vivenciado durante a pandemia da infecção pelo SARS-CoV-2 e que diversas condições podem confluir para novas crises sanitárias, sociais, econômicas e/ou políticas, os autores compreendem que a organização dos serviços mínimos na APS tornarse-á pauta recorrente nas unidades de saúde e que, por tanto, é recomendável que aquelas que ainda não possuem colegiados de gestão considerem estruturá-los, a fim de potencializar as capacidades gerenciais do centro de saúde e prover respostas ágeis e adaptadas às necessidades locais frente a períodos de disruptivos.

\section{Conclusão}

A APS, sendo o ponto de primeiro contato preferencial e coordenadora de cuidados dos usuários do sistema de saúde, é desafiada e afetada pelos momentos de instabilidades sociais, econômicas, políticas e sanitárias. Nestes cenários, as unidades de saúde precisam definir quais serviços serão ofertados à população, levando em conta as novas demandas que emergem durante as crises, bem como as atividades já existentes que precisam ser mantidas e os recursos disponíveis. Desta forma, este artigo buscou estabelecer e desambiguar as definições dos conceitos de atividades essenciais e serviços mínimos de forma ampla e generalizada e sua aplicação ao contexto da APS, utilizando-se da vivência ímpar proporcionada pela pandemia da covid-19 em 2020 em uma UBS na cidade de Florianópolis.

É importante destacar que a experiência aqui descrita foi vivenciada em um município cuja organização destaca-se pela presença de práticas inovadoras e bem-sucedidas na mudança do modelo assistencial na última década e uma elevada cobertura populacional pela ESF, e que a reprodução desta experiência está intimamente ligada à estrutura da APS nos municípios.

Adentrando-se 2021 com a experiência acumulada de um ano de enfrentamento da pandemia, é momento de se estudar os dados gerados com o intuito de se analisar as diversas estratégias adotadas pelos serviços de APS, comparando-se o impacto destas na saúde da população, como a cobertura vacinal, as taxas de internações por condições sensíveis à APS, o aumento dos casos de sofrimento psíquico e de violências, entre outros.

É esperado que novas crises acometam a sociedade e, por tanto, faz-se mister que a APS se instrumente para que possa se manter resolutiva, reorganizando-se de forma ágil e 
coordenada frente a estes períodos desafiadores, devendo preparar-se ainda nos momentos de "normalidade", apropriando-se das necessidades da população sob seus cuidados através da vigilância territorial e da garantia de acesso. Também é importante que os serviços de APS desenvolvam e incorporem novas tecnologias, para que consigam responder às novas demandas de saúde emergentes durante cenários atípicos, buscando formas de otimizar as suas atividades e processos, sejam eles assistenciais ou organizacionais, como os exemplos do uso das tecnologias da informação e comunicação e a prática da gestão colegiada, respectivamente.

Dada a necessidade de maior conhecimento em relação às questões gerenciais da atenção primária, é imperioso que se estabeleçam linhas de pesquisas sobre os serviços mínimos na APS em contextos de crise, tema cujo presente trabalho se propôs a fomentar as discussões iniciais.

\section{Contribuição autoral}

C. N. Souza participou da concepção, planejamento, análise, interpretação, redação do trabalho e aprovou o formato final; F. Manzini participou da concepção, planejamento, interpretação, redação do trabalho e aprovou o formato final; e S. N. Leite participou na interpretação, redação do trabalho e aprovou o formato final.

\section{Referências}

1. Paim J, Travassos C, Almeida C, Bahia L, Macinko J. The Brazilian health system: history, advances, and challenges. Lancet. 2011 May 21;377(9779):1778-97. doi: https://doi.org/10.1016/S0140-6736(11)60054-8.

2. Watkins DA, Yamey G, Schäferhoff M, Adeyi O, Alleyne G, Alwan A, et al. Alma-Ata at 40 years: reflections from the Lancet Commission on Investing in Health. Lancet. 2018 Oct 20;392(10156):1434-60. https://doi.org/10.1016/S0140-6736(18)32389-4.

3. World Health Organization. WHO announces covid-19 outbreak a pandemic. Copenhagen: WHO Regional Office for Europe; 2020 Mar 12 [citado 02 ago. 2020]. Disponível em: https://www.euro.who.int/en/health-topics/health-emergencies/coronavirus-covid19/news/news/2020/3/who-announces-covid-19-outbreak-a-pandemic

4. McLuhan M, Gordon WT, Lamberti E, Scheffel-Dunand D. The Gutenberg galaxy: the making of typographic man. Toronto: University of Toronto Press; 2011.

5. World Health Organization, United Nations Children's Fund. Declaration of Astana. Global Conference on Primary Health Care. Astana (Kazakhstan): [WHO]; 2018. 
6. Ministério da Saúde (BR). Carteira de serviços da atenção primária à saúde (CaSAPS): versão profissionais de saúde e gestores: resumida. [Brasília, DF]: MS; 2019.

7. Abrucio FL, Grin EJ, Franzese C, Segatto CI, Couto CG. Combate à covid-19 sob o federalismo bolsonarista: um caso de descoordenação intergovernamental. Rev Adm Publica. 2020;54(4):663-77. https://doi.org/10.1590/0034-761220200354

8. Ministério da Saúde (BR). Portaria n. 467, de 20 de março de 2020. Dispõe, em caráter excepcional e temporário, sobre as ações de telemedicina, com o objetivo de regulamentar e operacionalizar as medidas de enfrentamento da emergência de saúde pública de importância internacional previstas no art. 3. da Lei n. 13.979, de 6 de fevereiro de 2020, decorrente da epidemia de covid-19. Diário Oficial da União. 23 mar. 2020 [citado 2 ago. 2021];56-B(Seção 1, Extra):1. Disponível em: https://www.in.gov.br/en/web/dou/-/portaria-n-467-de-20-de-marco-de-2020-249312996

9. Giovanella L, Martufi V, Mendoza DCR, MHM, Bousquat AEM, Pereira RAG, et al. The contribution of primary health care in the SUS network to face Covid-19. Scielo Preprints [Preprint]. 2020 [citado 2 ago. 2021]. Disponível em: https://preprints.scielo.org/index.php/scielo/preprint/view/1286.

10. Cabré MT. La terminología hoy: concepciones, tendencias y aplicaciones. Cienc Inform. 1995 [citado 2 ago. 2021];24(3). Disponível em: http://revista.ibict.br/ciinf/article/view/567.

11. Portugal. Parecer do Conselho Consultivo da PGR: P001001989. [Lisboa]: 1989 [citado 2 ago. 2021]. Disponível em: http://www.dgsi.pt/pgrp.nsf//A30CB4A837ABF37C80258296005235A0

12. Brasil. Decreto n. 10.282, de 20 de março de 2020. Regulamenta a Lei n. 13.979 , de 6 de fevereiro de 2020, para definir os serviços públicos e as atividades essenciais. Diário Oficial da União. 20 mar. 2020 [citado 03 ago. 2021]. Edição extra G. Disponível em: http://www.planalto.gov.br/ccivil_03/_ato2019-2022/2020/decreto/D10282.htm.

13. World Health Organization. Role of primary care in the covid-19 response. Manila: WHO; 2020.

14. Starfield B. Is primary care essential?. Lancet. 1994 Oct 22;344(8930):1129-33. https://doi.org/10.1016/S0140-6736(94)90634-3.

15. Batista DCA. Greve nos serviços essenciais: breve análise comparada entre Brasil e Portugal. Coimbra: Universidade de Coimbra; 2015. 
16. Oliveira MAC, Pereira IC. Atributos essenciais da atenção primária e a estratégia saúde da família. Rev Bras Enferm. 2013;66:158-64. https://doi.org/10.1590/S003471672013000700020 .

17. Conselho Nacional de Secretários de Saúde. Atendimento da rede de atenção à saúde durante a pandemia COVID-19. [Brasília, DF]: CONASS; 2020.

18. Mendes EV. O lado oculto de uma pandemia: a terceira onda da covid-19 ou o paciente invisível. [Brasília, DF: CONASS]; 2020.

19. Ministério da Saúde (BR). Portaria n. 221, de 17 de abril de 2008. [Lista brasileira de internações por condições sensíveis à atenção primária] [Internet]. Brasília, DF: MS; 2008 [citado 3 ago. 2021]. Disponível em: https://bvsms.saude.gov.br/bvs/saudelegis/sas/2008/prt0221_17_04_2008.html.

20. Frateschi MS, Cardoso CL. Práticas em saúde mental na atenção primária à saúde. Psico (Porto Alegre). 2016;47(2):159-68. http://dx.doi.org/10.15448/1980-8623.2016.2.22024.

21. Lacerda JT, Botelho LJ, Colussi CF. Planejamento na atenção básica [Recurso eletrônico]. Florianópolis: UFSC; 2016 [citado 3 ago. 2021. Disponível em: https://www.google.com/url?sa $=t \& r c t=j \& q=\& e s r c=s \&$ source $=$ web\&cd $=\& c a d=r j a \& u a c t=$ 8\&ved=2ahUKEwic8tPV2pXyAhWIFrkGHVCvB9YQFnoECAQQAw\&url=https\%3A\%2F\%2Fun asus.ufsc.br\%2Fatencaobasica\%2Ffiles\%2F2017\%2F10\%2FPlanejamento-naAten\%25C3\%25A7\%25C3\%25A3o-B\%25C3\%25A1sica-ilovepdfcompressed.pdf\&usg=AOvVaw1bXTIDMjpy-Jk22_1ZJtd8.

22. Nodari $\mathrm{CH}$, Camargo ME, Olea PM, Dorion ECH, Claus SM. Configuração das práticas de inovação na atenção primária à saúde: estudo de caso. Cienc Saude Colet. 2015;20(10):3073-86. https://doi.org/10.1590/1413-812320152010.03262015.

23. Silveira JPM, Zonta R. Experiência de reorganização da APS para o enfrentamento da COVID-19 em Florianópolis. APS Rev. 2020;2(2):91-6. https://doi.org/10.14295/aps.v2i2.122.

24. Hart JT. The inverse care law. Lancet. 1971 Feb 27;1(7696):405-12. https://doi.org/10.1016/S0140-6736(71)92410-X.

25. André AM, Ciampone MHT. Competências para a gestão de Unidades Básicas de Saúde: percepção do gestor. Rev Esc Enferm USP. 2007;41(n. esp.):835-40. https://doi.org/10.1590/S0080-62342007000500017. 
26. Cecilio LCO. Colegiados de gestão em serviços de saúde: um estudo empírico. Cad Saude Publica. 2010;26(3):557-66. https://doi.org/10.1590/S0102-311X2010000300013.

Caique Nogueira de Souza | https://orcid.org/0000-0003-0719-279X http://lattes.cnpq.br/7267391740853028

Fernanda Manzini | https://orcid.org/0000-0002-3047-4632

http://lattes.cnpq.br/1917157621712374

Silvana Nair Leite | https://orcid.org/0000-0002-5258-9684

http://lattes.cnpq.br/9922706294578800 